factory for maintenance and growth. Lysin and tryptophan are both present. Other experiments by Osborne and Mendel indicate that lysin and tryptophan in the diet are both necessary for growth. If lysin be present without tryptophan maintenance is secured, but not growth. The writers found lysin $\mathrm{N}$ in both corn and cotton seed. The qualitative tests for tryptophan were positive. Unfortunately the determination of tryptophan by hydrolysis with acids has never yielded satisfactory results, it being thought that the tryptophan complex is broken down.

$$
\text { I } 7 \text { (I049) }
$$

\title{
The distribution of blood in shock.
}

\section{By H. H. Janeway and Holmes C. Jacksor.}

\section{[From the Department of Physiology, University and Bellevue Hospital Medical College.]}

In a recent communication we have shown that the essential factor of shock is a disturbance in the normal distribution of the blood. This disturbance is of such a character that the normal quota upon the arterial side of the circulation is diminished and this diminution is maintained so that, as a consequence, even after the original cause of the disturbed distribution of the blood, whether of a mechanical, toxic or inhibitory nature, is removed the abnormal diminution of the blood upon the arterial side of the circulation not only persists but, in fatal cases, progresses from local peripheral causes alone until death occurs.

The reality and nature of these local factors in the production of shock is made clearest in that form of shock which is produced by mechanical means alone, because in shock created in this manner no other factor can enter except the consequences of a primary disturbance of the normal distribution of the blood.

The mechanical means which we adopted for the production of shock was that used for the reduction of blood pressure when testing out the shock-producing effect of trauma to the peripheral sensory nerves. It consisted in partially occluding the inferior vena cava within the chest by passing a thread around the vein and drawing out the two ends through the incision in the wall of 
the thorax. By the degree of tension exerted on this thread, the amount of blood passing from the veins to the arterial side of the circulation could be accurately controlled and, in consequence, the blood pressure.

It was found that a two-hour period of reduction of the arterial blood pressure to from 30 to $40 \mathrm{~mm}$. of $\mathrm{Hg}$ was, with few exceptions, fatal within the next eighteen hours, even though there might be a rise of arterial blood pressure to nearly the normal height for a period of three to four hours after the release of the ligature around the inferior vena cava. Manometric tracings of the systemic and the portal venous pressures showed an immediate rise in the venous blood pressure within these veins, following the occlusion of the inferior vena cava. As the experiment continued, there was a gradual fall in the pressures.

Volumetric tracings of the intestinal loops showed regularly a fall in the volume of the intestines during the period of occlusion. In the majority of the experiments, for a short time during the beginning of the period of occlusion there was a rise in the volume of the intestines. This was succeeded by a steady fall, which was maintained until a terminal stage when a very extreme condition of shock was reached, in which the volume of the intestine would show a small terminal increase.

Volumetric tracings of the liver, however, showed a consistent increase in the volume of this organ to a late stage in shock. The volume of the liver showed an increase relative to that of the intestines. It would decrease in size at the time of the terminal increase in volume of the intestines; and we attributed the terminal increase in volume of the intestines as being due simply to a draining away of the blood sequestrated in the liver by tissues in the intestines absolutely devoid of tone.

During the period when the progressive fall in the volume of the intestines is well instituted, the volume of the intestines and also of the liver shows a marked dependence upon the arterial blood pressure; whereas in the normal animal at the start of the experiment a tightening of the ligature around the inferior vena cava and the production of a rise in this manner of the portal venous pressure caused an increase also in the volume of the intestines. Later in the experiment, a similarly produced increase 
of portal venous pressure showed a diminution of the volume of the intestines. In other words, the volume of the intestines during shock varied directly with arterial blood pressure, irrespective of increase or decrease in the portal venous pressure.

This, so to speak, inverse relation of the volume of the intestine can only be explained by recognizing that under such conditions of lowered blood pressure so little blood is supplied by the arterial side of the circulation that the effect of still further cutting down the arterial supply produces a greater effect upon the volume of the gut than the increased intravenous pressure. It must mean that the combined sectional area of the arterioles is already small.

These results confirm by another method of experimentation the conclusions of Mann, and of Morison and Hooker, and the failure in shock of venopressor mechanism, previously described by Henderson. Although we do not agree with Henderson's explanation of the cause of the failure of the venopressor mechanism, yet we entirely agree with the importance which he has ascribed to this mechanism. Our own experiments not only confirm the failure of the venopressor mechanism in shock but indicate the extent of this failure and the factors upon which it depends. We can see no other factor which can be responsible for the continued fall of blood pressure, after the period of partial occlusion of the inferior vena cava, than merely the effect of the mechanical distention of increased intravascular pressure upon the capillaries and small venules of regions draining into the inferior vena cava, and believe that this conclusion is emphasized by the comparatively high level to which the arterial blood pressure returns after the release of the ligature.

Of all parts of the vascular system, the capillaries and small venules are least capable of resisting the effects of increased intravascular pressure. The combined sectional area of the capillaries is very much greater than that of either the arterioles or the venules. Any increase in the capillary bed, even to a small extent, must, therefore, profoundly affect the general blood pressure. It is certain that during the period of occlusion of the vena cava the low blood pressure is due to the retention of blood within these vessels. In our experiments there is certainly no special sequestration in the vessels of the small intestine. The 
experiments indicate rather that there is a special sequestration of the blood in the capillaries of the liver, but further that the increase in size of the liver, coupled with the diminution in the size of the intestines, cannot alone account for the diminished arterial blood pressure.

The crucial factor concerns the fact that after the relief of the obstruction to the flow of blood through the inferior vena cava there occurs, even after a more or less complete restoration of the arterial blood pressure, a progressive fall until death. This progressive fall can only be a consequence of some change in the walls of the vessels primarily affected by the occlusion of the inferior vena cava, namely, the capillaries and small venules. It is quite obvious, inasmuch as the total amount of blood circulating is a constant quantity, that an over filling of the vessels on the venous side of the most peripherally situated vessels possessing a muscular wall must be accompanied by diminished total sectional area of the vessels on the arterial side of the last vessels possessing power of active resistance to the volume of blood contained in them. All experiments indicate that the lumen of the smaller arteries and arterioles are diminished. The pressure laws of vaso-motor adjustment under conditions of reduced arterial blood pressure, the comments of Henderson, the findings of Muns, the results of the perfusion experiments of Morison and Hooker, all indicate that the arterioles and smaller arteries possess a diminished lumen in shock. We have demonstrated that there is at least no greater response to the injections of adrenalin in shock than before shock has been induced. This fact explains the failure of any great increase in volume of the combined intraabdominal organs in shock.

In other words, our experiments of mechanically induced shock indicate that a mechanical distension of a certain degree and length of time is able to cause an alteration of the normally present contractility of the capillaries; in other words, of their tone, in virtue of which they lose the power of emptying the increased quantity of blood which they contain through the heart into the arterial side of the circulation, a power which they still preserve after either shorter periods of over-distention or distention of lesser degrees. 
In mechanical shock induced by obstruction to the venous return to the heart, the over-distention is due to a draining back of the blood within the capillaries. In inhibitory shock, the overdistention is due to the sudden discharge of blood at high pressure into the capillaries. In either case, the effect upon the capillary wall is the same, and shock would therefore consist in a general displacement of what might be termed a critical quantity of blood from the arterial to the capillary and venous side of the circulation, a displacement which is therefore accompanied by comparatively insignificant volumetric changes and dependent largely upon a loss of a normally existing tone in the walls of the small venules and capillaries.

\section{8 (1050)}

On the application of the Kjeldahl method of nitrogen determination to serological problems. (Preliminary note.)

BY ByroN W. Barshinger. (By invitation.)

[From the Department of Bacteriology and Immunity, Leland Stanford Jr. University.]

Practically no variation in the nitrogen content of rabbit serum (centrifuged) is produced by the usual variations in temperature at which the blood sample (sealed) is kept during the initial separation of the serum. Sera separated in the incubator, ice chest and at room temperature are identical, within the limits of the experimental error.

An increase of as much as 100 per cent. in nitrogen content may be brought about by increasing the length of time the serum is allowed to stand in contact with the clot. This increase is most rapid and most pronounced in samples kept at incubator temperature.

Variations as great as 40 per cent. may be observed in different samples removed from the same rabbit at the same bleeding. Small consecutive samples drawn at five-minute intervals may show differences as great as io per cent.

Variations as great as 40 per cent. above or below the average may be observed in sera of normal rabbits of the same age, size and breed, kept and bled under identical conditions. 